\title{
Downregulation of FoxM1 sensitizes nasopharyngeal carcinoma cells to cisplatin via inhibition of MRN-ATM-mediated DNA repair
}

\author{
Dandan $\mathrm{Li}^{1,2, \#}$, Lin $\mathrm{Ye}^{3, \#}$, Yue Lei ${ }^{2}$, Jie Wan ${ }^{1,2} \mathcal{E}$ Hongyan Chen ${ }^{1, *}$ \\ ${ }^{1}$ Department of Otolaryngology, The First Affiliated Hospital of Chongqing Medical University, Chongqing 40016, ${ }^{2}$ Laboratory Research \\ Center, The First Affiliated Hospital of Chongqing Medical University, Chongqing 40016, ${ }^{3}$ Department of cardiothoracic surgery, The First \\ Affiliated Hospital of Chongqing Medical University, Chongqing 400016, China
}

\begin{abstract}
Chemoresistance is the primary obstacle in the treatment of locally advanced and metastatic nasopharyngeal carcinoma (NPC). Recent evidence suggests that the transcription factor forkhead box M1 (FoxM1) is involved in chemoresistance. Our group previously confirmed that FoxM1 is overexpressed in NPC. In this study, we investigated the role of FoxM1 in cisplatin resistance of the cell lines 5-8F and HONE-1 and explored its possible mechanism. Our results showed that FoxM1 and NBS1 were both overexpressed in NPC tissues based on data from the GSE cohort (GSE12452). Then, we measured FoxM1 levels in NPC cells and found FoxM1 was overexpressed in NPC cell lines and could be stimulated by cisplatin. MTT and clonogenic assays, flow cytometry, $\gamma \mathrm{H} 2 \mathrm{AX}$ immunofluorescence, qRT-PCR, and western blotting revealed that downregulation of FoxM1 sensitized NPC cells to cisplatin and reduced the repair of cisplatin-induced DNA double-strand breaks via inhibition of the MRN (MRE11-RAD50-NBS1)-ATM axis, which might be related to the ability of FoxM1 to regulate NBS1. Subsequently, we demonstrated that enhanced sensitivity of FoxM1 knockdown cells could be reduced by overexpression of NBS1. Taken together, our data demonstrate that downregulation of FoxM1 could improve the sensitivity of NPC cells to cisplatin through inhibition of MRN-ATM-mediated DNA repair, which could be related to FoxM1-dependent regulation of NBS1. [BMB Reports 2019; 52(3): 208-213]
\end{abstract}

*Corresponding author. Tel: +86-23-89012758; Fax: +86-2389012758; E-mail: clara213@163.com

${ }^{\#}$ These authors contributed equally to this work.

https://doi.org/10.5483/BMBRep.2019.52.3.249

Received 27 October 2018, Revised 13 November 2018 , Accepted 10 January 2019

Keywords: Cisplatin, FoxM1, MRN-ATM axis, Nasopharyngeal carcinoma, Resistance

\section{INTRODUCTION}

Nasopharyngeal carcinoma (NPC) is a malignancy with a high incidence rate in select geographic and ethnic populations (1). Presently, chemoradiotherapy is advocated for the treatment of locally advanced and metastatic NPC, and cisplatin is commonly used for chemotherapy $(2,3)$. However, following primary treatment, one-third of patients will relapse with either locoregional recurrence or distant metastases $(4,5)$. Therefore, the exploration of genes related to NPC drug resistance as therapeutic targets is urgently needed.

Cisplatin-induced inter-strand adducts can lead to DNA double-strand breaks (DSBs) $(6,7)$. Responding to DSBs, cells will perform a series of reactions referred to as the DNA damage response (DDR), which serves to activate an intricate network of signaling pathways to trigger cell cycle arrest and DNA repair, and resulting in senescence and apoptosis if the lesions are irreparable (7). DSBs are primarily repaired by homologous recombination $(\mathrm{HR})$ and non-homologous end joining (NHEJ) (8). NBS1 (also known as NBN) plays an important role in the recruitment of Mrell/Rad50 to the nucleus, where it forms the MRN (MRE11-RAD50-NBS1) complex, which binds damaged DNA directly and initiates HR-dependent repair (9). The MRN complex also works to recruit and activate ataxia-telangiectasia mutated (ATM), a vital kinase in the DDR signaling network. The activation of ATM regulates hundreds of substrates concerned with cell cycle checkpoint control, DNA repair, and apoptosis $(10,11)$.

Forkhead box M1 (FoxM1) is a transcription factor involved in a series of normal biological processes as well as the development and tumorigenesis of various cancers $(12,13)$. Recently, FoxM1 has been reported to play a critical role in chemoresistance by regulating DNA repair mechanisms (14, 15). In this study, we explored the relationship between FoxM1 expression and cisplatin resistance in NPC for the first time. Our results indicate that FoxM1 knockdown cells were susceptible to DSBs following treatment with cisplatin, and FoxM1 might play an important role in DSB repair via inhibition of the MRN-ATM axis. 


\section{RESULTS}

\section{FoxM1 and NBS1 are overexpressed in head and neck squamous carcinoma (HNSC), and especially NPC}

We employed the UALCAN database (http://ualcan.path. uab.edu/index.html) to determine the expression level of FoxM1 and NBS1 in HNSC. As shown in Supplementary Fig. S1A and S1C, FoxM1 and NBS1 were both overexpressed in HNSC samples compared to normal samples $(P=1.62 \times$ $10^{-12}, \mathrm{P}=1.11 \times 10^{-16}$, respectively). Furthermore, expression of FoxM1 and tumor grade in HNSC was inversely correlated with overall survival $(\mathrm{P}=0.039)$ (Supplementary Fig. S1B). Next, we used the GEPIA database (http://gepia.cancerpku.cn/) to analyze the correlation between FoxM1 and NBS1 in HNSC. FoxM1 expression was positively correlated with NBS1 expression in HNSC tissues $(\mathrm{R}=0.4, \mathrm{P}<0.001)$ (Supplementary Fig. S1D). NPC is a squamous carcinoma that originates in the nasopharynx epithelium (16). Based on the GSE cohort (GSE12452) from GPL570: [HG-U133_Plus_2] Affymetrix Human Genome U133 Plus 2.0 Array (https:// www-ncbi-nlm-nih-gov-cd.vtrus.net/geo/query/acc.cgi?acc = GPL570), we determined that expression of FoxM1 and NBS1 were both prominently higher in NPC compared with normal tissues $(\mathrm{P}<0.001, \mathrm{P}<0.01$, respectively) (Fig. 1A). Thus, we determined FoxM1 mRNA expression in normal and NPC cell lines by RT-PCR. FoxM1 mRNA levels in the normal cell line NP69 were significantly lower than the NPC

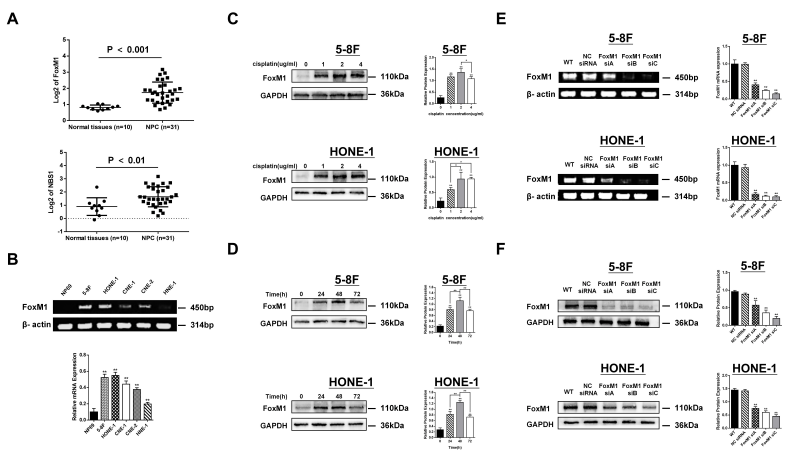

Fig. 1. FoxM1 and NBS1 are overexpressed in nasopharyngeal carcinoma (NPC) and FoxM1 expression was induced by cisplatin treatment in NPC cells. (A) GSE cohort indicating expression of FoxM1 and NBS1 in NPC and normal tissues. (B) FoxM1 mRNA expression in the NPC cell lines 5-8F, HONE-1, CNE-1, CNE-2, and HNE-1 and the normal cell line NP69 were detected by RT-PCR. (C) 5-8F and HONE-1 cells were treated with 0, 1, 2, and $4 \mu \mathrm{g} / \mathrm{ml}$ cisplatin. After $48 \mathrm{~h}$, FoxM1 protein levels were determined by western blotting. (D) $5-8 \mathrm{~F}$ and HONE-1 cells were treated with $1 \mu \mathrm{g} / \mathrm{ml}$ cisplatin for $0,24,48$, and $72 \mathrm{~h}$. FoxM1 protein levels were determined by western blotting. 5-8F and HONE-1 cells were transfected with three FoxM1 siRNAs and NC siRNA. (E) FoxM1 mRNA levels were determined by RT-PCR and qRT-PCR. (F) FoxM1 protein levels were determined by western blotting. $* P<0.05 ; * * P<0.01$. cell lines 5-8F, HONE-1, CNE-1, CNE-2, and HNE-1 (Fig. 1B), indicating that FoxM1 is indeed overexpressed in NPC.

\section{FoxM1 expression was induced by cisplatin treatment in NPC cells}

To further assess the characteristics of FoxM1 expression in NPC cells following cisplatin treatment, we treated 5-8F and HONE-1 cells with increasing concentrations of cisplatin. Fig. 1C shows that FoxM1 protein expression was increased following cisplatin treatment. Then, we measured FoxM1 expression levels in cells treated with cisplatin for different lengths of time and found FoxM1 expression was highest at 48 h (Fig. 1D). Thus, our results indicated that FoxM1 expression could be induced by treatment with cisplatin.

\section{Silencing of FoxM1 expression enhanced the sensitivity of NPC cells to cisplatin}

To investigate whether overexpression of FoxM1 is related to cisplatin resistance, we silenced FoxM1 using three FoxM1 small interfering RNAs (siRNAs) and found that siRNA-C resulted in the greatest knockdown of FoxM1 (Fig. 1E, 1F). Then, using MTT and clonogenic assays, flow cytometry, and western blotting to measure proliferation, cell cycle distribution, and apoptosis in 5-8F and HONE-1 cells, we demonstrated that knockdown of FoxM1 resulted in lower cell survival rate and increased sensitivity to cisplatin, as evidenced by a comparison of IC50 values between NC (Negative Control) siRNA and FoxM1 siRNA-treated cells (2.24

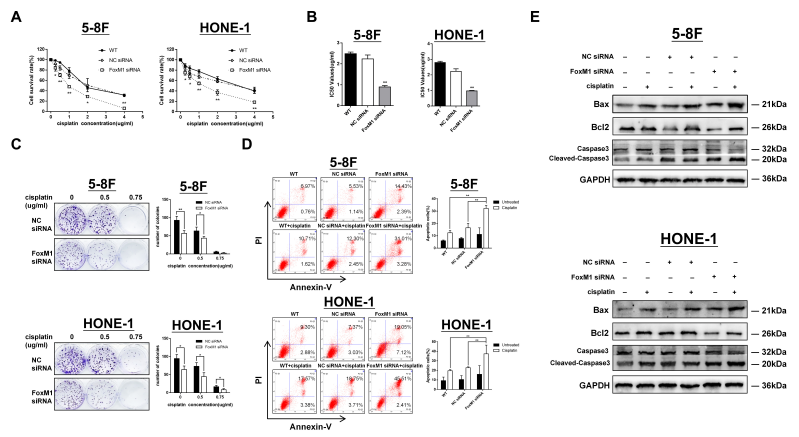

Fig. 2. Silencing of FoxM1 expression enhanced the sensitivity of NPC cells to cisplatin. (A) Cell survival rate of 5-8F and HONE-1 cells treated with NC or FoxM1 siRNA and increasing concentrations of cisplatin were tested by MTT assay. (B) IC50 values of cisplatin were analyzed by Probit regression analysis. (C) Colony-formation ability of 5-8F and HONE-1 cells treated with $\mathrm{NC}$ or FoxM1 siRNA and the indicated concentrations of cisplatin were tested by clonogenic assay. NPC cells were transfected with FoxM1 or NC siRNA. After $48 \mathrm{~h}$, cells were treated with $1 \mu \mathrm{g} / \mathrm{ml}$ cisplatin for another $48 \mathrm{~h}$. (D) Apoptosis of 5-8F and HONE-1 cells was measured by flow cytometry. (E) Expression levels of the apoptotic markers $\mathrm{Bax}, \mathrm{Bcl} 2$, caspase-3, and cleaved caspase-3 of $5-8 \mathrm{~F}$ and HONE-1 cells were measured by western blotting. *P $<0.05 ; * * P 0.01$. 
$\pm 0.19 \mu \mathrm{g} / \mathrm{ml}$ vs $0.9 \pm 0.07 \mu \mathrm{g} / \mathrm{ml}$ in $5-8 \mathrm{~F}$ cells, $2.23 \pm 0.16$ $\mu \mathrm{g} / \mathrm{ml}$ vs $0.97 \pm 0.03 \mu \mathrm{g} / \mathrm{ml}$ in HONE-1 cells) (Fig. 2A, 2B). Cells transfected with FoxM1 siRNA resulted in fewer colonies than cells transfected with NC siRNA at different concentrations of cisplatin in 5-8F and HONE-1 cells (Fig. 2C). Our results suggested a cell cycle arrest at G0/G1 phase in 5-8F cells (Supplementary Fig. S1E); cells treated with FoxM1 siRNA and cisplatin presented a higher apoptotic rate compared with cells treated with NC siRNA and cisplatin or cells treated with cisplatin only (Fig. 2D). It is also worth noting that the expression of Bax and cleaved caspase-3 was significantly increased while $\mathrm{Bcl} 2$ expression was decreased in cells treated with both cisplatin and FoxM1 siRNA (Fig. 2E and Supplementary Fig. S1F), which is consistent with the change in apoptotic rate. Collectively, downregulation of FoxM1 enhanced the sensitivity of NPC cells to cisplatin.

\section{Silencing of FoxM1 caused a deficiency in DNA repair via inhibition of the MRN-ATM axis in NPC cells}

$\gamma \mathrm{H} 2 \mathrm{AX}$ is considered the most reliable biomarker of DSBs (17). To explore the function of FoxM1 in DNA repair in NPC cells, we used $\gamma \mathrm{H} 2 \mathrm{AX}$ immunofluorescence to measure DSBs in 5-8F and HONE-1 cells. Cells treated with FoxM1 siRNA and cisplatin exhibited a greater number of $\gamma \mathrm{H} 2 \mathrm{AX}$ foci in comparison to cells treated with NC siRNA and cisplatin or cisplatin only (Fig. 3A). To further investigate how FoxM1 modulates DNA repair in NPC, we performed qRT-PCR and western blotting to determine the expression of genes associated with DNA repair in 5-8F and HONE-1 cells. At the

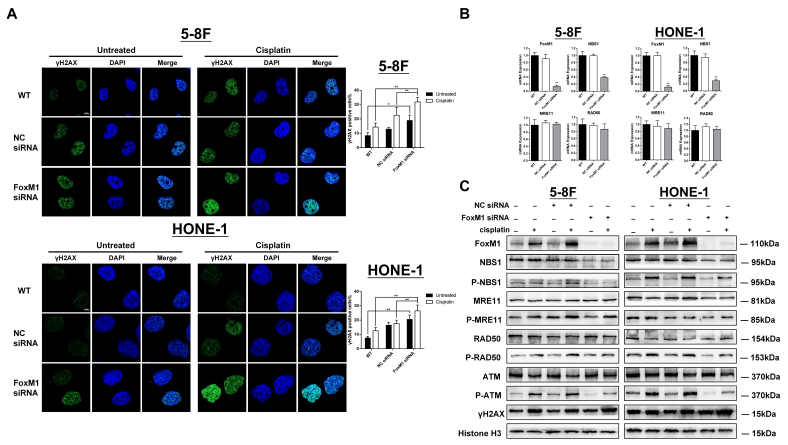

Fig. 3. Silencing of FoxM1 caused DNA repair deficiency via inhibition of the MRN-ATM axis in NPC cells. (A) $\gamma \mathrm{H} 2 \mathrm{AX}$ foci of $5-8 \mathrm{~F}$ and HONE-1 cells were detected by $\gamma \mathrm{H} 2 \mathrm{AX}$ immunofluorescence. Scale bars of representative images represent $5 \mu \mathrm{m}$. Cells with greater than 10 foci were counted as positive. The percentage of $\gamma \mathrm{H} 2 \mathrm{AX}$-positive cells was plotted. 5-8F and HONE-1 cells were transfected with NC or FoxM1 siRNA. (B) At $24 \mathrm{~h}$ after transfection, mRNA levels of FoxM1, NBS1, MRE11, and RAD50 were measured by qRT-PCR. (C) At $48 \mathrm{~h}$ after transfection, cells were treated with $1 \mu \mathrm{g} / \mathrm{ml}$ cisplatin for another 48 h. Protein levels of FoxM1, NBS1, P-NBS1, MRE11, P-MRE11, RAD50, P-RAD50, ATM, P-ATM, and $\gamma \mathrm{H} 2 \mathrm{AX}$ were measured by western blotting. ${ }^{*} \mathrm{P}<0.05 ; * * P<0.01$.
mRNA level, knockdown of FoxM1 decreased NBS1 expression, while the expression of MRE11 and RAD50 remained almost unchanged (Fig. 3B). At the protein level, knockdown of FoxM1 reduced NBS1, P-NBS1 (Phosphorylated NBS1), and P-ATM (Phosphorylated ATM), and increased $\gamma \mathrm{H} 2 \mathrm{AX}$ in a cisplatin-independent manner (Fig. 3C and Supplementary Fig. S2A). These changes indicate that silencing of FoxM1 could cause a deficiency in DNA repair via inhibition of the MRN-ATM axis, which might be related to the ability of FoxM1 to regulate NBS1.

\section{Overexpression of NBS1 induced cisplatin resistance of FoxM1 knockdown cells}

To verify that downregulation of NBS1 in FoxM1 knockdown cells is responsible for the increased sensitivity to cisplatin, we overexpressed NBS1 in 5-8F and HONE-1 cell lines (Fig. 4A). MTT and flow cytometry assays were used to determine whether the increased sensitivity of NPC cells to cisplatin by silencing FoxM1 could be reduced by overexpression of NBS1. Cells treated with thiostrepton (FoxM1 inhibitor) and pcDNA3.1-NBS1 presented higher cell survival rate compared with cells treated with thiostrepton and pcDNA3.1 (Fig. 4B). Furthermore, the sensitivity of FoxM1 knockdown cells to cisplatin was reduced by the overexpression of NBS1, as evidenced by a comparison of IC50 values between the thiostrepton and pcDNA3.1 group and the thiostrepton and pcDNA3.1-NBS1 group $(0.97 \pm 0.2 \mu \mathrm{g} / \mathrm{ml}$ vs $1.86 \pm 0.03$

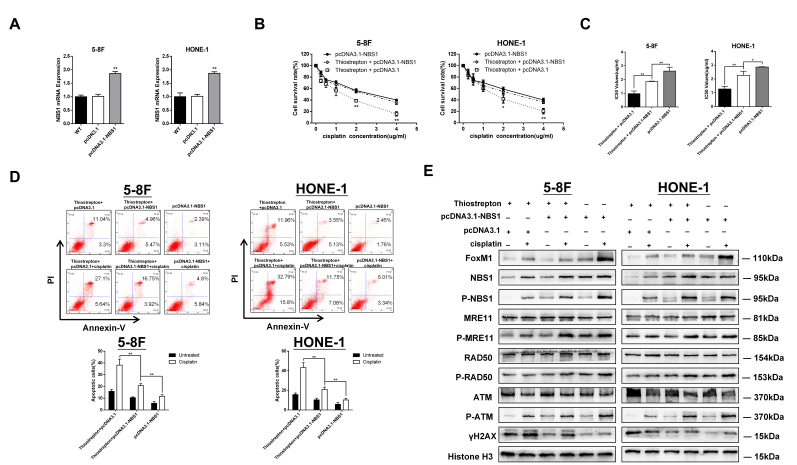

Fig. 4. Overexpression of NBS1 induced cisplatin resistance of FoxM1 knockdown cells. (A) 5-8F and HONE-1 cells were transfected with pcDNA3.1 or pcDNA3.1-NBS1. FoxM1 mRNA levels were determined by qRT-PCR. (B) Cell survival rate of 5-8F and HONE-1 cells treated with thiostrepton and pcDNA3.1, thiostrepton and pcDNA3.1-NBS1, or pCDNA3.1-NBS1 only and increasing concentrations of cisplatin were tested by MTT assay. (C) IC50 values of cisplatin were analyzed by Probit regression analysis. NPC cells were treated with thiostrepton and pcDNA3.1, thiostrepton and pcDNA3.1-NBS1, or pcDNA3.1-NBS1 only. After $48 \mathrm{~h}$, cells were treated with $1 \mu \mathrm{g} / \mathrm{ml}$ cisplatin for another $48 \mathrm{~h}$. (D) Apoptosis of 5-8F and HONE-1 cells was measured by flow cytometry. (E) Protein levels of FoxM1, NBS1, P-NBS1, MRE11, P-MRE11, RAD50, P-RAD50, ATM, P-ATM, and $\gamma \mathrm{H} 2 \mathrm{AX}$ were measured by western blotting. ${ }^{*} P<0.05 ; * * P<0.01$. 
$\mu \mathrm{g} / \mathrm{ml}$ in $5-8 \mathrm{~F}$ cells, $1.28 \pm 0.2 \mu \mathrm{g} / \mathrm{ml}$ vs $2.27 \pm 0.29 \mu \mathrm{g} / \mathrm{ml}$ in HONE-1 cells) (Fig. 4C). Moreover, overexpression of NBS1 in FoxM1 knockdown cells decreased cisplatin-induced apoptosis (Fig. 4D). We next used western blotting to assess proteins associated with DNA repair in 5-8F and HONE-1 cells. Downregulation of NBS1, P-NBS1, and P-ATM induced by silencing FoxM1 could be disrupted by overexpression of NBS1. Consistent with this, overexpression of NBS1 decreased $\gamma \mathrm{H} 2 \mathrm{AX}$ expression in NPC cells (Fig. 4E and Supplementary Fig. S2B). These data demonstrated that overexpressing NBS1 could induce resistance of FoxM1 knockdown cells to cisplatin, suggesting NBS1 signaling was involved in FoxM1 and NPC chemosensitivity.

\section{DISCUSSION}

The majority of patients present with locally advanced or metastatic NPC when newly diagnosed (18). Despite treatment, the prognosis of late stage NPC patients remains poor (19); thus, the development of targeted therapies for NPC is important.

Our group previously showed that FoxM1 is overexpressed in NPC and has been linked to proliferation, apoptosis, invasion, migration, and angiogenesis (20, 21). However, whether FoxM1 is related to the chemoresistance of NPC has remained elusive. In this study, we investigated the role of FoxM1 in the cisplatin resistance of the cell lines $5-8 \mathrm{~F}$ and HONE-1 and explored its possible mechanisms. With the help of databases, we determined that FoxM1 and NBS1 were overexpressed in HNSC and positively correlated. Next, we determined that FoxM1 and NBS1 were both overexpressed in NPC tissues based on data from the GSE cohort. Then, we measured FoxM1 levels in NPC cells and found that FoxM1 was overexpressed in NPC cell lines and could be stimulated by cisplatin. Using MTT and clonogenic assays, flow cytometry, $\gamma \mathrm{H} 2 \mathrm{AX}$ immunofluorescence, qRT-PCR, and western blotting, we determined that silencing of FoxM1 expression enhanced sensitivity of NPC cells to cisplatin and reduced the repair of cisplatin-induced DSBs via inhibition of the MRN-ATM axis. Moreover, the increased sensitivity of FoxM1 knockdown cells could be reduced by overexpressing NBS1. This evidence suggested that downregulation of FoxM1 could inhibit the MRN-ATM axis via regulation of NBS1, and subsequently improved the sensitivity of NPC cells to cisplatin.

The MRN-ATM axis is involved in HR, which is the most precise mechanism for the repair of $\operatorname{DSBs}(8,22)$. NBS1-disrupted cells have been shown to exhibit a marked reduction in HR events, suggesting NBS1 is required for HR (23). Our results suggested that NBS1 might be a target of FoxM1; however, further studies are needed to confirm whether FoxM1 directly targets NBS1 and which region of the NBS1 promoter FoxM1 binds in NPC. In addition to NBS1, FoxM1 has been shown to target a wide range of DNA repair related genes, including XRCC1, RAD51, BRIP1, EXO1, RFC4,
Csk1, and Skp2. These genes are not only involved in HR repair, but also in NHEJ, base-excision, and mismatch repair (24). These data argue that FoxM1 is indispensable for DNA repair, and that knockdown of FoxM1 can largely abrogate DNA repair and accumulate DNA damage.

Several clinical findings have revealed that the DNA repair status of tumor cells is related to patient response to cisplatin treatment (25). In cancer cells, increased repair or tolerance of DNA damage may contribute to chemoresistance (26). We found that FoxM1 was overexpressed in NPC and could be stimulated by cisplatin. Given that FoxM1 is essential for DNA repair, its overexpression may increase DNA repair and accelerate cisplatin resistance.

In conclusion, we demonstrate that knockdown of FoxM1 enhanced cisplatin sensitivity, which could be mediated by inhibition of the MRN-ATM pathway via regulation of NBS1. To increase the anti-tumor effects of cisplatin, further studies investigating the effects of cisplatin combined with FoxM1-targeted therapeutic drugs for the treatment of NPC are warranted and may be useful in minimizing cisplatin resistance in NPC.

\section{MATERIALS AND METHODS}

\section{Cell lines and chemical compounds}

NPC cell lines 5-8F, HONE-1, CNE-1, CNE-2, and HNE-1 and the normal cell line NP69 were obtained from the American Type Culture Collection (Manassas, VA, USA). All cell lines were cultured in RPMI-1640 medium (Sigma-Aldrich, St. Louis, MO, USA) supplemented with $10 \%$ fetal bovine serum (PAN-Biotech, Germany), $100 \mu \mathrm{g} / \mathrm{ml}$ streptomycin, and 100 $\mathrm{U} / \mathrm{ml}$ penicillin. Cells were maintained in a humid atmosphere with $5 \% \quad \mathrm{CO}_{2}$ at $37^{\circ} \mathrm{C}$. Thiostrepton (Sigma, USA) was dissolved in DMSO (dimethylsulfoxide). As previously described, cells were treated with $8 \mu \mathrm{M}$ thiostrepton for $48 \mathrm{~h}$ (27), and then cell survival rate and apoptosis were detected by MTT and flow cytometry assays respectively.

\section{SiRNA transfection}

siRNA duplexes were purchased from GenePharma (Shanghai, China). Cells were transfected with siRNA by RNAi-Mate reagent (GenePharma) according to the manufacturer's instructions. siRNA sequences are listed in Supplementary Table 1.

\section{Expression vector construction and transient transfections} pcDNA3.1-NBS1 was generated as previously described (28) and the sequence was verified. $5-8 \mathrm{~F}$ and HONE-1 cells were transfected with pcDNA3.1 or pcDNA3.1-NBS1 using Lipofectamine 2000 (Invitrogen, Carlsbad, CA, USA) according to the manufacturer's instructions. 


\section{Semi-quantitative RT-PCR (RT-PCR) and quantitative real-time PCR (qRT-PCR)}

Total RNA was extracted using TRIzol reagent (Takara, Japan) and complementary DNA (cDNA) was synthesized using the Primer Script RT reagent kit (Takara) according to the manufacturer's instructions. For RT-PCR, cDNA was mixed with Premix Taq (Takara) and amplified using a Bio-Rad system (Bio-Rad Laboratories, Hercules, CA, USA). The amplification cycling program was $94^{\circ} \mathrm{C}$ for $3 \mathrm{~min}, 30$ cycles of $94^{\circ} \mathrm{C}$ for $30 \mathrm{~s}$ and $58^{\circ} \mathrm{C}$ for $30 \mathrm{~s}$, and $72^{\circ} \mathrm{C}$ for $1 \mathrm{~min}$. The amplification products were detected by agarose gel electrophoresis and images were analyzed using a Bio-Rad Molecular Imager. QRT-PCR was performed and analyzed as described previously (27). Primers for RT-PCR and qRT-PCR were synthesized by Takara and the sequences are listed in Supplementary Tables 2 and 3.

\section{Western blotting}

Nucleoprotein was extracted using the Nuclear and Cytoplasmic Protein Extraction Kit (Beyotime Biotechnology, China) according to the manufacturer's instructions. Total protein was extracted using radioimmunoprecipitation buffer supplemented with the protease inhibitor PMSF. Western blotting was performed as described previously (27). Each blot was repeated in three separate experiments. Band intensities were measured using Fusion software and the data are presented as relative protein levels normalized to GAPDH or histone $\mathrm{H} 3$. The antibodies used in this study were as follows: FoxM1 and P-RAD50 (Cell Signaling Technology, Danvers, MA, USA); Bax, Bcl2, GAPDH, NBS1, P-NBS1, MRE11, P-MRE11, RAD50, ATM, P-ATM, and $\gamma \mathrm{H} 2 \mathrm{AX}$ (Abcam, Cambridge, UK); and caspase-3, cleaved caspase-3, and histone $\mathrm{H} 3$ (Wanleibio, China).

\section{MTT assay}

At $24 \mathrm{~h}$ after transfection, cells were resuspended and seeded in 96-well plates at a density of $5 \times 10^{3}$ cells/well and incubated for another $24 \mathrm{~h}$. Cisplatin was added to the media. After $48 \mathrm{~h}, 20 \mu \mathrm{l}$ of methyl thiazolyl tetrazolium (MTT) (Sigma-Aldrich) solution was added to each well and incubated for another $4 \mathrm{~h}$. Then, the liquid in the wells was removed and dimethyl sulfoxide was added (Sigma-Aldrich). After $10 \mathrm{~min}$, the absorbance was measured at $570 \mathrm{~nm}$ using a microplate reader (Tecan, Mannedorf, Switzerland).

\section{Clonogenic assay}

Cells transfected with FoxM1 and NC siRNAs were treated with cisplatin for $2 \mathrm{~h}$. Then, the cells were resuspended and seeded in 6-well plates at a density of 500 cells/well. After incubation for 2 weeks, cells were fixed with $4 \%$ paraformaldehyde for $10 \mathrm{~min}$ and stained with crystal violet for $15 \mathrm{~min}$. Colonies were counted if the number of cells was greater than or equal to 50 .
Flow cytometry for cell cycle distribution and apoptotic rate At $48 \mathrm{~h}$ after transfection, 5-8F cells were collected to analyze cell cycle distribution by flow cytometry. 5-8F and HONE-1 cells were treated with $1 \mu \mathrm{g} / \mathrm{ml}$ cisplatin for another $48 \mathrm{~h}$, and cells were stained with annexin V-FITC and propidium iodide to detect apoptosis by flow cytometry. Flow cytometry was performed as described previously (29).

\section{$\gamma \mathrm{H} 2 \mathrm{AX}$ immunofluorescence}

$5-8 \mathrm{~F}$ and HONE-1 cells were transfected with NC or FoxM1 siRNAs. At $48 \mathrm{~h}$ after transfection, cells were treated with 1 $\mu \mathrm{g} / \mathrm{ml}$ cisplatin for another 48 h. $\gamma \mathrm{H} 2 \mathrm{AX}$ immunofluorescence staining was performed as described previously (14). Representative images were captured using a confocal microscope. For statistical analysis, cells with greater than 10 foci were considered positive according to standard procedure (30). More than 500 cells were assessed in each group, and the experiment was performed in triplicate.

\section{Statistical analysis}

SPSS 22.0 and GraphPad Prism 6.01 software were used for statistical analysis. IC50 values of cisplatin were analyzed by Probit regression analysis. The differences among groups were analyzed by one-way analysis of variance. P-values less than 0.05 were considered statistically significant: ${ }^{*} \mathrm{P}<0.05$; $* * \mathrm{P}$ $<0.01$.

\section{ACKNOWLEDGEMENTS}

This work was supported by Natural Science Foundation of China (grant numbers: 81272980).

\section{CONFLICTS OF INTEREST}

The authors have no conflicting interests.

\section{REFERENCES}

1. Torre LA, Bray F, Siegel RL, Ferlay J, Lortet-Tieulent J and Jemal A (2015) Global cancer statistics, 2012. CA Cancer J Clin 65, 87-108

2. Al-Sarraf M, LeBlanc M, Giri PG et al (1998) Chemoradiotherapy versus radiotherapy in patients with advanced nasopharyngeal cancer: phase III randomized Intergroup study 0099. J Clin Oncol 16, 1310-1317

3. Yoshizaki T, Kondo S, Murono S et al (2015) Progress and controversy for the role of chemotherapy in nasopharyngeal carcinoma. Jpn J Clin Oncol 45, 244-247

4. Lee AW, Sze WM, Au JS et al (2005) Treatment results for nasopharyngeal carcinoma in the modern era: the Hong Kong experience. Int J Radiat Oncol Biol Phys 61, 1107-1116

5. Lee AW, Poon YF, Foo W et al (1992) Retrospective analysis of 5037 patients with nasopharyngeal carcinoma treated during 1976-1985: overall survival and patterns of 
failure. Int J Radiat Oncol Biol Phys 23, 261-270

6. Galluzzi L, Senovilla L, Vitale I et al (2012) Molecular mechanisms of cisplatin resistance. Oncogene 31, 1869-1883

7. Goldstein M and Kastan MB (2015) The DNA damage response: implications for tumor responses to radiation and chemotherapy. Annu Rev Med 66, 129-143

8. Paull TT and Lee JH (2005) The Mre11/Rad50/Nbs1 complex and its role as a DNA double-strand break sensor for ATM. Cell Cycle 4, 737-740

9. Frappart PO, Tong WM, Demuth I et al (2005) An essential function for NBS1 in the prevention of ataxia and cerebellar defects. Nat Med 11, 538-544

10. So S, Davis AJ and Chen DJ (2009) Autophosphorylation at serine 1981 stabilizes ATM at DNA damage sites. J Cell Biol 187, 977-990

11. Lamarche BJ, Orazio NI and Weitzman MD (2010) The MRN complex in double-strand break repair and telomere maintenance. FEBS Lett 584, 3682-3695

12. Laoukili J, Stahl M and Medema RH (2007) FoxM1: at the crossroads of ageing and cancer. Biochim Biophys Acta $1775,92-102$

13. Koo CY, Muir KW and Lam EW (2012) FOXM1: From cancer initiation to progression and treatment. Biochim Biophys Acta 1819, 28-37

14. Zhou J, Wang Y, Wang Y et al (2014) FOXM1 modulates cisplatin sensitivity by regulating EXO1 in ovarian cancer. PLoS One 9, e96989

15. Park YY, Jung SY, Jennings NB et al (2012) FOXM1 mediates Dox resistance in breast cancer by enhancing DNA repair. Carcinogenesis 33, 1843-1853

16. Chua MLK, Wee JTS, Hui EP and Chan ATC (2016) Nasopharyngeal carcinoma. Lancet 387, 1012-1024

17. Kuo LJ and Yang LX (2008) Gamma-H2AX - a novel biomarker for DNA double-strand breaks. In Vivo 22, 305-309

18. Wang KMsd, Chen ZMsd, Long LMsd et al (2018) iTRAQ-based quantitative proteomic analysis of differentially expressed proteins in chemoresistant nasopharyngeal carcinoma. Cancer Biol Ther 19, 1-16

19. Bensouda Y, Kaikani W, Ahbeddou N et al (2011) Treatment for metastatic nasopharyngeal carcinoma. Eur Ann Otorhinolaryngol Head Neck Dis 128, 79-85
20. Yu C, Chen L, Yie L et al (2015) Targeting FoxM1 inhibits proliferation, invasion and migration of nasopharyngeal carcinoma through the epithelialto-mesenchymal transition pathway. Oncol Rep 33, 2402-2410

21. Jiang L, Wang $P$ and Chen $H$ (2014) Overexpression of FOXM1 is associated with metastases of nasopharyngeal carcinoma. Ups J Med Sci 119, 324-332

22. San Filippo J, Sung P and Klein H (2008) Mechanism of eukaryotic homologous recombination. Annu Rev Biochem 77, 229-257

23. Tauchi H, Kobayashi J, Morishima K et al (2002) Nbs1 is essential for DNA repair by homologous recombination in higher vertebrate cells. Nature 420, 93-98

24. Nestal de Moraes G, Bella L, Zona S, Burton MJ and Lam EW (2016) Insights into a critical role of the FOXO3a-FOXM1 axis in DNA damage response and genotoxic drug resistance. Curr Drug Targets 17, 164-177

25. Rocha CRR, Silva MM, Quinet A, Cabral-Neto JB and Menck CFM (2018) DNA repair pathways and cisplatin resistance: an intimate relationship. Clinics (Sao Paulo) 73, e478s

26. Salehan MR and Morse HR (2013) DNA damage repair and tolerance: a role in chemotherapeutic drug resistance. Br J Biomed Sci 70, 31-40

27. Jiang L, Wang $P$, Chen $L$ and Chen H (2014) Down-regulation of FoxM1 by thiostrepton or small interfering RNA inhibits proliferation, transformation ability and angiogenesis, and induces apoptosis of nasopharyngeal carcinoma cells. Int J Clin Exp Pathol 7, 5450-5460

28. Zheng J, Zhang C, Jiang L et al (2011) Functional NBS1 polymorphism is associated with occurrence and advanced disease status of nasopharyngeal carcinoma. Mol Carcinog 50, 689-696

29. Jiang L, Wu X, Wang P et al (2015) Targeting FoxM1 by thiostrepton inhibits growth and induces apoptosis of laryngeal squamous cell carcinoma. J Cancer Res Clin Oncol 141, 971-981

30. Ziebarth AJ, Nowsheen S, Steg AD et al (2013) Endoglin (CD105) contributes to platinum resistance and is a target for tumor-specific therapy in epithelial ovarian cancer. Clin Cancer Res 19, 170-182 